\title{
Les médecines complémentaires dans les hôpitaux universitaires
}

\author{
Jean Martin \\ Dr med., membre de la rédaction
}

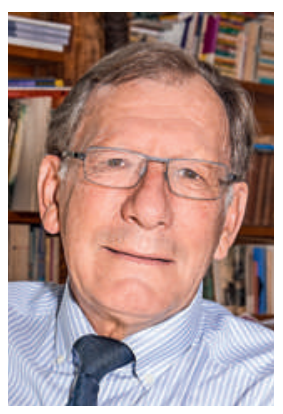

RTS Un, émission $36.9^{\circ}$ 27 janvier 2016

Le terme "complémentaires» paraît préférable à celui de «alternatives».

3 Graz B. Les médecines complémentaires Dépasser les clivages. Lausanne: Presses polytechniques et universitaires romandes; 2012.

4 Souvenir personnel d'il y a 40 ans: un de nos professeurs de médecine, interpelé à propos de médecines complémentaires, a répondu «Il ne faut pas étudier scientifiquement ces méthodes parce qu'elles ne sont pas scientifiques...» On croit rêver. Quel contresens, quelle myopie chez un enseignant enfoncé dans, mais aussi limité par, ce qu'il voit comme la seule science possible.
Sous le titre Les médecines complémentaires sont entrées dans les hôpitaux universitaires, bien intéressante émission du magazine santé de la télévision romande [1], sur la présence, plutôt discrète mais réelle, des médecines complémentaires [2] dans les grands hôpitaux. Entre autres le CHUV de Lausanne: impressionnant de voir des professeurs estimés dire comment des moyens différents de ceux enseignés en faculté ont beaucoup aidé des patients, là où les techniques orthodoxes ne réussissaient pas. Par exemple: l'ostéopathie dans les douleurs vertébrales de la femme enceinte; les évolutions réjouissantes de grands brûlés suite à l'appel à des profanes qui "ont le secret" (domaine où on reste spécialement démuni pour apporter une explication rationnelle quelconque!) - l'hypnose s'étant aussi avérée fort utile dans les soins aux brûlés. Une séquence est tournée à l'Hôpital cantonal de St-Gall. Récemment, la presse a évoqué à plusieurs reprises l'emploi de techniques de méditation de type pleine conscience, dans le suivi de dépressifs notamment, à Genève (HUG) et dans d'autres sites. Les enquêtes faites montrent qu'il arrive à la moitié de nos concitoyens de faire appel à des méthodes «autres». Mon propos ici n'est pas de prêcher en leur faveur, mais de relever qu'il y a dans ce qui précède des décisions judicieuses d'ouverture de la part de cliniciens et enseignants qui permettent, voire jugent bienvenus, l'intervention de pratiques/praticiens non-orthodoxes dans leurs services. En fait, c'est aussi une preuve d'objectivité! On pourra ici sursauter et dire que je suis incohérent, puisque précisément ces méthodes n'ont souvent pas (encore) démontré leur efficacité dans nos termes scientifiques à nous. Mais je parle ici d'objectivité de bon sens, de tous les jours. Ces méthodes apportent des bénéfices, ont observé nos confrères, elles aident les malades, parfois beaucoup.

Aujourd'hui, chacun admet j'espère que, si la formation donnée dans les facultés est bonne et nécessaire, elle ne saurait être toute la vérité. Et chacun se souvient de mots de grands esprits dans le sens de «Plus j'apprends, plus je réalise combien je suis encore ignorant.» J'ai décrit il y a longtemps déjà ma surprise devant le fait suivant: quand des patients que leur médecin allopathe connaît bien lui parlent de leur dernier voyage, de leur réussite ou malheur en affaires, du film qu'ils ont vu, ce praticien les croit, les considère comme capables de discernement.
Mais quand les mêmes personnes disent avoir été grandement aidées par l'homéopathie, par exemple, alors il s'agit pour certains confrères d'autosuggestion, d'illusion... Cherchez l'erreur. Je n'ai pas de lien avec l'homéopathie mais j'ai entendu trop de témoignages crédibles pour décréter qu'elle ne sert à rien du tout. Le rapport Médecines complémentaires de novembre 2010 de la Faculté lausannoise et du CHUV, donnant suite à des démarches antérieures de sensibilisation, ne dit pas autre chose: «On ne doit pas négliger le fait que diverses approches sont ressenties comme bénéfiques par les personnes qui y ont recours".

Ce rapport est à la base des développements actuels à Lausanne: une commission des médecines complémentaires y est instituée, qui supervise le Centre de médecine intégrative et complémentaire - CEMIC (par médecine intégrative il faut entendre une démarche de combinaison de la médecine conventionnelle avec les méthodes autres). A noter la publication d'un ouvrage de synthèse sur le sujet par un membre senior du CEMIC [3]. Aux facultés de Berne et Zurich, on s'est préoccupé de médecine complémentaire depuis une vingtaine d'années et les deux ont aujourd'hui un institut de ce nom (avec à Zurich aussi le mot «intégrative»).

ll faut bien sûr ne pas céder à des modes mais il n'est plus tenable, comme certains le faisaient dogmatiquement, de vouer ces méthodes aux gémonies [4]. D’autant plus que les rapides progrès de la science (voir les neurosciences) pourraient bien démontrer pourquoi des approches aujourd'hui incompréhensibles fonctionnent. De plus, il y a lieu de voir qu'on est là au sein d'évolutions qui vont dans le sens d'une médecine plus consciente de ce qu'elle est située dans une société donnée et ne vit pas «dans sa bulle»; une médecine plus attentive à l'autonomie et à l'opinion du patient et qui met l'accent sur le relationnel (qu'on pense aux Medical Humanities dans la formation - ou en clinique aux soins palliatifs, qui font d'ailleurs appel à des méthodes complémentaires, «soft»). Clairement, il faut écarter l'antagonisme d'antan où la médecine académique ne voulait pas entendre parler de toute technique qui lui était extérieure. L'avenir doit être sous le signe du respect (ce qui n'empêche pas l'esprit critique) et de la collaboration chaque fois que les faits le justifient - ou pour le moins de la coexistence pacifique. 